\title{
A self-assembled monolayer-assisted surface microfabrication and release technique
}

\author{
B.J. Kim ${ }^{\mathrm{a}, 1}$, M. Liebau ${ }^{\mathrm{b}}$, J. Huskens ${ }^{\mathrm{b}}$, D.N. Reinhoudt ${ }^{\mathrm{b}}$, J. Brugger ${ }^{\mathrm{a}, *}$ \\ ${ }^{a}$ Nanolink Strategic Research Orientation, MESA ${ }^{+}$Research Institute, University of Twente, P.O. Box 217, \\ 7500 AE Enschede, The Netherlands \\ ${ }^{\mathrm{b}}$ Supramolecular Chemistry and Technology Group, MESA ${ }^{+}$Research Institute, University of Twente, P.O. Box 217, \\ 7500 AE Enschede, The Netherlands
}

\begin{abstract}
This paper describes a method of thin film and MEMS processing which uses self-assembled monolayers as ultra-thin organic surface coating to enable a simple removal of microfabricated devices off the surface without wet chemical etching. A 1.5-nm thick self-assembled monolayer of dodecyltrichlorosilane reduces the adhesion between the $\mathrm{SiO}_{2}$ substrate surface and a 100-nm thick evaporated aluminum film. A 100- $\mu \mathrm{m}$ thick layer of photoplastic SU-8, which is spun and structured by lithography and development on top of the monolayer/aluminum sandwich layer, can be mechanically lifted off the surface with the aluminum layer. The organic monolayer provides enough stability for the microfabrication process including photoresist spinning and thermal steps. The aluminum film has a surface roughness of less than $1 \mathrm{~nm}$ rms as measured by AFM. Photolithographic microstructuring of the aluminum film prior to the photoplastic process allows for transparent embedded bottom-side metal electrodes. As first application example, molded nanoprobes for scanning near-field optical microscopy, has been demonstrated using this technique. (c) 2001 Elsevier Science B.V. All rights reserved.
\end{abstract}

Keywords: Self-assembled monolayer; Dry release; Demolding; Photoplastic SU-8; MEMS/NEMS

\section{Introduction}

A common problem of surface microfabricated micro-electro-mechanical systems (MEMS) is the post-process releasing or demolding of the structures from the underlying substrate. As example, silicon wafers often serve as support or mold to replicate a microstructure in built-on layers such as, for instance, nanoprobes and cantilevers for atomic force microscopes [1]. In many cases the surface structure can only be freed from the mold by etching the wafer underneath completely away.

\footnotetext{
*Corresponding author. Tel.: + 31-53-489-5652; fax: + 31-53-489-2575.

E-mail address: j.p.brugger@el.utwente.nl (J. Brugger).

${ }^{1}$ Present address: Institute of Industrial Science, University of Tokyo, CIRMM (Center for International Research on MicroMechatronics), 4-6-1, Komaba, Meguro-ku, Tokyo 153-8505, Japan.
} 
Alternatively, nanoprobes fabricated by sacrificial layer etching have been demonstrated which enables to reuse the high resolution, and hence cost-intensive master mold for multiple replications [2]. However, the use of chemical sacrificial layer etching has several drawbacks: first it induces contamination, and second, the necessary thickness of the sacrificial layer for efficient underetching (typically $\sim 1 \mu \mathrm{m}$ ) prevents accurate replication of the structure at the nanometer scale.

Functionalizing silicon surfaces with organic monolayers is already used for instance to reduce stiction in movable micromachines [3], or for their dry releasing by using an organic sacrificial layer via plasma etching [4].

In the present work we demonstrate the use of self-assembled monolayers (SAMs) for a different method of microstructure fabrication. We exploit differences in surface adhesion forces between thin deposited and microstructured layers to separate them without the need for additional chemical process steps. The mechanical release method allows for instance surface structures to be lifted off a surface mold with a structured metal layer attached to it, such as required for instance for nanoprobes for scanning near-field optical microscopy (SNOM). Furthermore, patterning the metal film can enable a double-side electrode configuration.

\section{Fabrication details}

\subsection{Surface modification with self-assembled monolayer}

A silicon dioxide surface is functionalized by a self-assembled monolayer (SAM) composed of organosilicon derivatives $\mathrm{R}_{n} \mathrm{SiX}_{3-n}$, where $\mathrm{X}$ is chloride or alkoxy. They are known to form ordered monolayers on hydroxylated surfaces [5]. Immersing an oxidized silicon wafer into a solution of the organosilicon derivative forms the SAM. The main observation is a drastic change of the wettability of the surface. Clean silicon dioxide surfaces are hydrophilic (contact angle with water $0^{\circ}$ ), while surfaces coated with paraffin-type structures are hydrophobic (contact angle with water $>90^{\circ}$ ). The chemical reaction can be described as polycondensation between the $\mathrm{Si}-\mathrm{X}$ bond and the $\mathrm{OH}$-groups present on the surface, presumably coming from traces of water adsorbed on the wafer surface.

The reaction forms a network of molecules connecting them to the surface and to each other by covalent bonds. The kinetics of the monolayer formation has been studied using contact angle measurement [6]. An equilibrium was found after 3-min adsorption of dodecyltrichlorosilane (DTS). Other studies (thickness measurements) show that a plateau value of about $1.5 \mathrm{~nm}$ will be reached after $40 \mathrm{~min}$. SAMs of alkytrichlorosilanes are stable against detergents and organic solvents. They withstand also acidic solutions, whereas under basic conditions the monolayers will be deteriorated. Stability studies against thermal effects showed moderate changes up to $100^{\circ} \mathrm{C}$ for SAMs on aluminum [7].

In the present fabrication sequence, an oxidized $10-\mathrm{mm}$ silicon wafer was immersed in a 5-10-mM solution of DTS in dry toluene under a glove box. In specific cases the silicon surface has been structured previously by lithography and anisotropic wet etching using potassium hydroxide $(\mathrm{KOH})$ which formed inverted pyramids subsequently used as nanomolds for nanoprobes [8]. Because SAMs are only a few nanometer thick they are able to cover surface contours very conformal down to the molecular scale. This is crucial for accurate replication of the molds at the nanometer scale. 


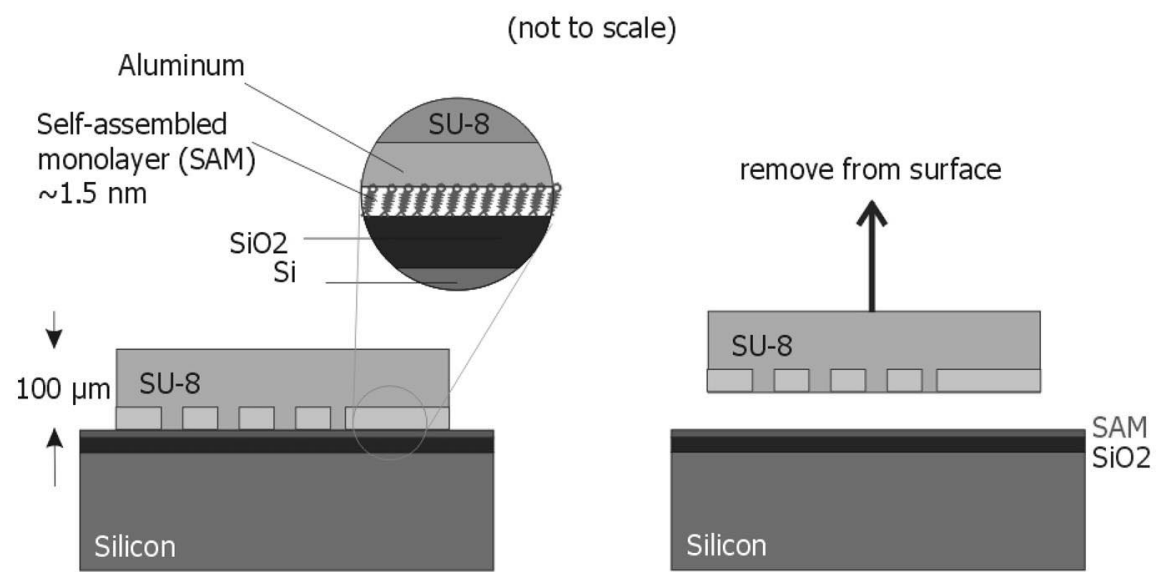

Fig. 1. Schematic representation of the sandwich layer and release process, showing the 1.5-nm thick self-assembled monolayer (SAM) on the oxidized silicon surface covered by a 100-nm thick evaporated and patterned Al layer, and finally spin-on structured photoplastic SU-8. Low adhesion between Al and the silicon dioxide allow to remove the plastic with the Al pattern.

\subsection{Al coating}

A $100-\mathrm{nm}$ thick aluminum (Al) film is then evaporated directly onto the SAM in a resistive evaporator at a base pressure of 6E-06 mbar. The distance between the evaporation source and the sample was $30 \mathrm{~cm}$ and the deposition time was 4-5 min. The Al layer is then patterned by lithography and wet etching. In some cases we evaporated the metal locally using microshadow masks [9] in order to avoid any chemical contamination, which are typically induced by the associated processes in lithography and etching steps.

\section{3. $S U-8$ spin-on process}

Then a 100- $\mu \mathrm{m}$ thick layer of photoplastic material (e.g. SU-8) is spun and patterned [10]. The resulting sandwich layer consisting of $\mathrm{SiO}_{2}, \mathrm{SAM}, \mathrm{Al}$ and $\mathrm{SU}-8$ is schematically shown in Fig. 1. The role of the SAM in this configuration is to form a 'nanocarpet' which reduces the adhesion between $\mathrm{Al}$ and $\mathrm{SiO}_{2}$ but which provides enough stability to support all subsequent micromachining process steps, such as multiple resist spinning, thermal steps, development and etching. We have chosen our layer configuration in such a way that the SU-8 structure at the end can be easily lifted off the surface together with the patterned metal layer attached to it.

\section{Results}

The dry lift-off method is demonstrated here in the case of two different microstructures: 


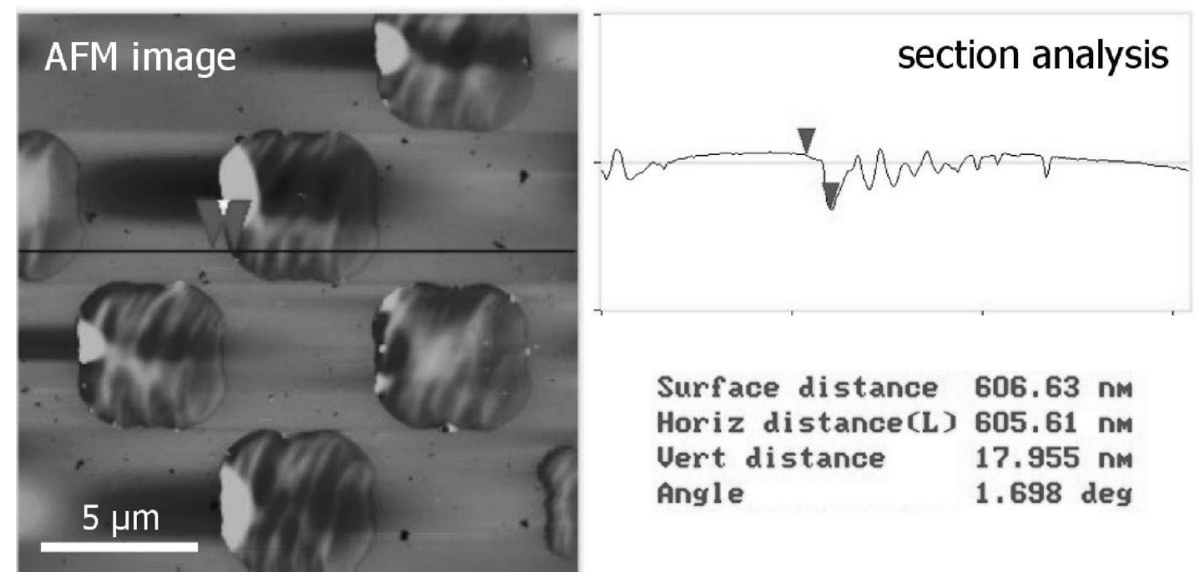

Fig. 2. AFM image showing a $15 \times 15-\mu \mathrm{m}$ section of the SU- 8 microstructure lifted off the $\mathrm{SiO}_{2} / \mathrm{SAM}_{\mathrm{S}}$ surface with the Al film (left). The Al film has been previously structured lithographically with arrays of 5- $\mu$ m wide openings. The surface corrugation of the $\mathrm{Al}$ is less than $1 \mathrm{~nm} \mathrm{rms}$, as shown by the cross sectional analysis of the single AFM line-scan over the 100 -nm thick Al pattern that is fully embedded in the SU-8. The height profile and surface corrugation of the SU-8 in the order of $\sim 10 \mathrm{~nm}$ is attributed to stress induced plastic deformation during the mechanical lift-off process.

\subsection{Photoplastic structure with embedded bottom-side metal pattern}

A block of photoplastic SU-8 is mechanically lifted off the surface with a 100-nm thick Al pattern attached to it, as shown in Figs. 1 and 2. Since no wet chemical release step is necessary, no contamination of the surfaces occurs which can be very important for the fabrication of micro/ nanoelectrode devices in (bio)chemical sensor systems.

A detailed view of the lifted surface is provided in Fig. 2. The atomic force microscope (AFM) image of a $15 \times 15 \mu \mathrm{m}$ shows a very flat Al surface with $0.8 \mathrm{~nm}$ rms surface roughness. Fig. 2 also shows a single AFM scan line of the same structure. The surface corrugation observed on the SU-8 section is attributed to the mechanical stress induced to the photoplastic material during the dry-lift-off step. We believe that this problem can be avoided by using an improved microassembly tool.

\subsection{Molded nanoprobe for scanning near-field optical microscopy (SNOM)}

The ability to unmold SU-8 structures with metal coating down to the nanometer scale has been successfully used to microfabricate a novel nanoprobe for scanning near-field optical microscopy (SNOM). The probe consists of a sharp light-guiding pyramidal tip (SU-8) covered by a light absorbing layer (Al). The process is adapted from a process for low-cost photoplastic AFM probes [2] and modified for the mass-fabrication of bright near-field optical probes (Fig. 3). In short, the $\mathrm{KOH}$-etched pyramidal mold is oxidation sharpened and then covered by the SAM following the process as described before. A 100-nm Al layer is evaporated, then two layers of SU-8 are spun (5 and $200 \mu \mathrm{m}$ ), exposed and developed to form a cup into which the optical fiber is micro-assembled and glued. Finally, the transparent nanoprobe is lifted off the mold with the Al coating as shown in Fig. 4. The aperture is formed using a focused ion beam (FIB) technique. The major asset of this 


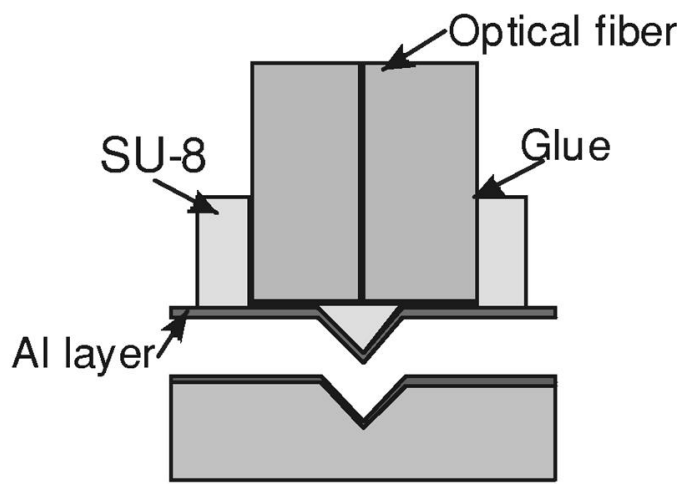

Fig. 3. Schematic illustration of photoplastic nanoprobe for scanning near-field optical microscopy (SNOM).

process is the smooth $\mathrm{Al}$ layer, allowing the aperture of the nanoprobe to be approached to the surface within a few nanometers.

\section{Conclusion and outlook}

This paper provides details on a recently developed technique to release micromachined photoplastic structures from the surface without any wet chemical sacrificial etching step. A ultra-thin, 1.5-nm thick, self-assembled monolayer of DTS was used to reduce the adhesion between the silicon dioxide surface and a Al/SU-8 microstructure. Simple mechanical lift-off allowed to fabricated embedded bottom-side metal pattern, and a novel nanoprobe for SNOM. We believe that, besides the presented

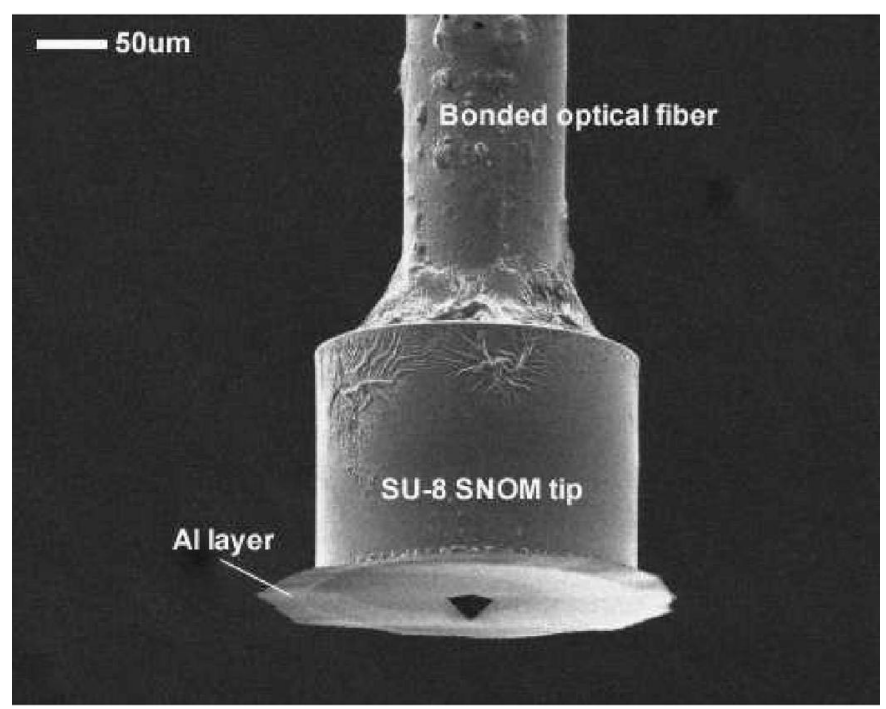

Fig. 4. Scanning electron micrograph of a fabricated photoplastic SU-8 nanoprobe for SNOM, micro-assembled and bonded to an optical fiber. 
examples, this technique has great potential for a series of surface micro- and nanofabricated devices where, e.g. double-side patterning of ultra-thin structures such as cantilevers and membranes is relevant. The highly conformal coverage of surface molds by $1.5-\mathrm{nm}$ thick SAMs in combination with a selective and 'non-wetting' surface releasing technique allows in particular for the fabrication of functionalized tips on the nanoscale, e.g. for advanced scanning probe systems.

In future, a more thorough understanding of the interface between the evaporated metal on the organic SAM is necessary to improve selectivity of the lift-off process. It is currently unknown what happens exactly to the SAM during mechanical lift-off. We assume that it remains on the $\mathrm{SiO}_{2}$ due to the strong covalent bonds. Further study will include various different material combinations, such as other metals and dielectrics. Also, it is important to investigate the damage made to the SAM by the deposited layer. Improved deposition techniques may allow to make contacts between metal and organic layers in order to fabricate micro/nanodevices and molecular electronic circuits.

\section{Acknowledgements}

The authors would like to thank the Optical Techniques Group of Professor Niek van Hulst for the collaboration with the SNOM probe, and all $\mathrm{MESA}^{+}$cleanroom staff for technical support of microfabrication. Financial support is acknowledged from $\mathrm{MESA}^{+}$Research Institute, University of Twente. One of the authors (ML) acknowledges support from the European Commission in the SPINUP project within the MELARI/NID framework.

\section{References}

[1] T.R. Albrecht, S. Akamine, T.E. Carver, C.F. Quate, J. Vac. Sci. Technol. A 8 (4) (1990) 3386-3396.

[2] G. Genolet, J. Brugger, M. Despont, U. Drechsler, P. Vettiger, N.F. de Rooij, D. Anselmetti, Soft, entirely photoplastic probes for scanning force microscopy, Rev. Sci. Instrum. 70 (5) (1999) 2398-2401.

[3] U. Srinivasan et al., Alkyltrichlorosilane-based self-assembled monolayer films for stiction reduction in silicon micromachines, J. Microelectromech. Syst. 2 (2) (1998) 252-260.

[4] H.-J. Suh, P. Bharathi, D.J. Beebe, J.S. Moore, Dendritic material as a dry-release sacrificial layer, J. Microelectromech. Syst. 9 (2) (2000) 1.

[5] J. Sagiv, J. Am. Chem. Soc. 102 (1980) 92.

[6] R. Maoz, J. Sagiv, J. Colloid Interface Sci. 100 (1984) 465.

[7] S.R. Cohen, R. Naaman, J. Sagiv, J. Phys. Chem. 90 (1986) 3054.

[8] B. Kim, J.W. Flamma, E.S. ten Have, M.F. Garcia-Parajo, N.F. van Hulst, J. Brugger, Molded photoplastic probes for near-field optical applications, J. Microsc. 202 (1) (2001) 16-21.

[9] J. Brugger, J.W. Berenschot, S. Kuiper, W. Nijdam, B. Otter, M. Elwenspoek, Resistless patterning of sub-micron structures by evaporation through nanostencils, Microelectron. Eng. 53 (2000) 403-405.

[10] M. Despont, H. Lorenz, N. Fahrni, J. Brugger, P. Renaud, P. Vettiger, High-aspect-ratio, ultrathick, negative-tone near-UV photoresist for MEMS applications: proceedings of MEMS conference 1997, Sensors Actuators A 64 (1998) 33. 